\title{
STRIPED QUANTUM HALL PHASES
}

\author{
FELIX VON OPPEN, ${ }^{(1)}$ BERTRAND I. HALPERIN, ${ }^{(2)}$ AND ADY STERN ${ }^{(3)}$ \\ (1) Institut für Theoretische Physik, Universität zu Köln, Zülpicher Str. 77, 50937 Köln, \\ Germany \\ (2) Physics Department, Harvard University, Cambridge, Massachusetts 02139, USA \\ (3) Department of Condensed Matter Physics, The Weizmann Institute of Science, 76100 \\ Rehovot, Israel
}

\begin{abstract}
Recent experiments seem to confirm predictions that interactions lead to charge density wave ground states in higher Landau levels. These new "correlated" ground states of the quantum Hall system manifest themselves for example in a strongly anisotropic resistivity tensor. We give a brief introduction and overview of this new and emerging field.
\end{abstract}

\section{Introduction}

While a large number of fractional quantized Hall states have been discovered in the lowest Landau level, such states become increasingly rare in higher Landau levels. Few quantized Hall states have been observed in the first Landau level (Landau level filling factor $2 \leq \nu<4$ ) and no such states have so far been found for filling factors $\nu \geq 4$. Correspondingly, the role of electron-electron interactions in this filling factor range has long remained almost uncharted territory.

This is now rapidly changing, following recent experiments by Lilly et al. 1 and by Du et al 3 on extremely clean two-dimensional electron systems (2DES) for Landau level (LL) filling factor $\nu>4$. The central observation is that the resistivity becomes strongly anisotropic close to half filling of the topmost Landau level in this filling factor range. This is believed to be a signature of a novel Coulomb-induced charge density wave ground state whose existence had been predicted by Fogler et al. . $^{3}$ and by Moessner and Chalker. 4 Unlike the Laughlin states, 5 these states can be partially understood within the Hartree-Fock (HF) approximation. In fact, it was already suggested at the end of the '70s by Fukuyama et al. 6 that the HF ground state of the lowest Landau level is a charge density wave (CDW). While in the lowest Landau level, the CDW ultimately turned out to be preempted by the Laughlin states, it seems to be more favorable in higher Landau levels.

Different ground states have been predicted depending on filling factor. 3 is Near half filling of higher Landau levels $(\nu \simeq N+1 / 2)$, the HF ground state should be a unidirectional charge density wave (UCDW). In this state, the filling factor alternates between stripes of filling factor $N$ and $N+1$. The period of the density modulation is of the order of the cyclotron radius $R_{c}$. Further away from half filling one expects a so-called bubble phase, in which clusters of the minority filling factor $(N$ or $N+1)$ order on a triangular lattice with characteristic length $R_{c}$. Very close to integer filling factors, a Wigner crystal phase should form.

The strongly anisotropic transport properties 1.8 near half filling of higher Landau levels are believed to be associated with the unidirectional charge density wave phase. In this paper we briefly review some of the recent work on these states. The experimental results are reviewed in Sec. 2. Sec. 3 focuses on theoretical devel-

submitted to World Scientific on November 12, 1999 
opments. In Sec. 3.1 we sketch early theoretical work which suggested that there exists an instability towards CDW formation. It is well known that Hartree-Fock calculations tend to overestimate the degree of ordering. Some insights into this can be gained from an analogy with two-dimensional liquid crystal phases which we review in Sec. 3.2. A focus of recent theoretical work are the transport properties of the UCDW states, as reviewed in Sec. 3.3. It has been shown under rather general assumptions that the conductivity tensor should satisfy non-trivial relations, which are independent of microscopic parameters 9 . 10 Finally we summarize and conclude in Sec. A by mentioning some open problems.

\section{Experiment}

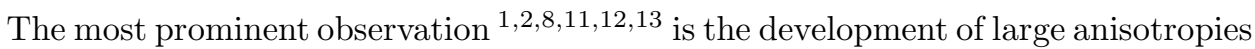
in the resistivity close to half filling of the topmost Landau level. Observation of this effect requires extremely clean samples and very low temperatures $(T<150 \mathrm{mK})$. The anisotropy develops even though the sample behaves essentially isotropically at very large and very low magnetic fields. The case for a new "correlated" ground state in this filling factor range is further strengthened by the observation that the width of the peak in the resistivity around half filling does not decrease with decreasing temperature. This is very different from what one would expect for the integer quantum Hall effect (QHE) plateau transition.

To date, the principal experimental features of these novel states are:

(a) The anisotropic resistivity has so far been observed near half filling of the topmost LL in the filling factor range $4 \leq \nu \leq 12$. The anisotropy is largest for $\nu=9 / 2$ and decreases monotonically with increasing LL index $N$.

(b) The principal axes of the anisotropic resistivity tensor seem to be consistently oriented along certain crystallographic axes of the GaAs crystal. The high-resistance direction is always along the $1 \overline{1} 0$ direction while the low-resistance direction is aligned with the 110 direction. 1 .

(c) The resistances in the two principal directions differ by up to several orders of magnitude in van-der-Pauw measurements.1.2 However, van-der-Pauw measurements significantly overestimate the anisotropy of the resistivity tensor (assuming that a local resistivity tensor is an appropriate description). 14.15 This is associated with the detailed current distribution in the device. Accordingly, Hall-bar measurements which should be free of this problem ${ }^{a}$ show a much smaller but still very significant anisotropy of the order of five.t

(d) The anisotropy usually appears more stable against temperature for filling factors corresponding to the lower spincomponent of each LL.

(e) A striking set of experiments11 12 revealed that even weak in-plane magnetic fields $B_{\|}$can have a strong effect on the anisotropic states (and on the even denominator fractional QHE state at $\nu=5 / 2$.) Applying the in-plane field in the low-resistance direction leads to an interchange of easy and hard directions for $B_{\|}$ of the order of $0.5 \mathrm{~T}$. In-plane fields in the high-resistance direction affect the measured resistivities in the upper spin component of each LL only very weakly, but suppress the anisotropy in the lower spin component.

${ }^{a}$ However, they currently measure the two diagonal resistivities on different samples.

submitted to World Scientific on November 12, 1999 
(f) Transport in the high-resistance direction is strongly non-linear with the differential resistance rising with increasing bias current. The change in resistivity with applied current is smooth, which would seem inconsistent with a depinning transition of the UCDY. The non-linearity is more pronounced in the lower spin component of each LL.]

(g) Intriguing reentrant integer QHE states have been found near quarter and

three quarter filling of the uppermost LL.16 The Hall resistance in these states is quantized at the value of the closest integer plateau. Current-voltage characteristics in this filling factor regime exhibit discontinuous and hysteretic behavipr. It has been suggested that this may be related to depinning of a CDW state.16

\section{Theory}

\subsection{Hartree-Fock calculations and numerical exact-diagonalization studies}

It turns out that essential features of the CDW ground states can be understood within the Hartree-Fock approximation (HFA). Most calculations assume that one can project to a single (partially filled) Landau level. This is natural for the lowest LL. For higher LLs, this relies on an effective interaction derived by Aleiner and Glazman 17 which includes the effects of screening by the lower (filled) LLs. This effective interaction turns out to be sufficiently weak compared to the Landau level spacing so that projection to a single LL should be a reasonable starting point.

The zero-temperature HF equations within the single-LL approximation have been studied in detail by Fogler et al. $3^{3}$ In particular, these authors compared various possible CDW ground states. In the vicinity of half filling of the topmost LL $(\nu \simeq N+1 / 2)$ they find that a unidirectional CDW state whose period is of the order of the cyclotron radius is most favorable. In this state, one-dimensional stripes of filling factor $N$ alternate with stripes of filling factor $N+1$. The analysis of this state is simplified by the fact that the exact HF eigenfunctions are still the usual Landau gauge wave functions for electrons in a magnetic field. All Landau gauge states with centers in the filling factor $N+1$ range are occupied, while those wavefunctions with centers in the filling factor $N$ region remain empty. Clearly, the relative modulation in the filling factor is larger than that of the electron density due to the finite width of the wavefunctions.

While CDW formation leads to a cost in Hartree energy due to the associated charge density modulation, this energy cost is more than offset by the gain in exchange energy due to the closer packing of the electrons. It turns out that this mechanism is particularly effective in higher LLs where the (Hermite-polynomial) wavefunctions have zeroes. In this case, there are wavevectors of the charge density wave for which the associated charge density modulation is particularly small. Essentially, this happen when a state centered in the middle of a filling factor $N+1$ region has its first side maximum in the filling factor $N$ region. It is for this reason that the CDW states are more favorable in higher LLs than in the lowest LL.

The bulk of this paper will be concerned with the unidirectional CDW. Further away from half filling, Fogler et al.3 predict a triangular CDW, termed bubble phase, consisting of clusters of minority filling factor ordering in a background of

submitted to World Scientific on November 12, 1999 
majority filling factor on a triangular lattice. Again, cluster size and period of the lattice are given by the cyclotron radius $R_{c}$. Very close to integer filling factors, the electrons (or holes) in the topmost Landau level are predicted to form a triangular Wigner crystal.

Moessner and Chalker 4 arrived at similar conclusions by deriving Landau theories for various CDW states and comparing their free energy. Following Fukuyama et al., 6 these authors expand the free energy in powers of the appropriate order parameter. This procedure is justified in the vicinity of the Hartree-Fock transition temperature $T_{c}^{h f}$. In addition, they show by diagrammatic arguments that the Hartree-Fock approximation becomes exact for the uniform phase in the limit of high Landau levels.

These conclusions have been partly supported by numerical exact diagonalization studies. In these calculations, systems of up to 12 electrons on a torus are diagonalized exactly and strong peaks indicating CDW ordering have been found in the wave vector dependence of the static density susceptibility and the equal-time density-density correlation function.

Motivated by the dramatic effect of in-plane magnetic fields $B_{\|}$in experiment, two groups 1812 extended the Hartree-Fock calculations to include the finite thickness of the 2DES and the resulting orbital effects of $B_{\|}$. While these calculations suggest that the influence of $B_{n}$ is sensitive to sample details, the perhaps more realistic of the two calculations 18 shows that in-plane fields can rotate the stripe pattern in the appropriate manner. These calculations do not explain the experimentally observed dependences on the spin of the LL.

\subsection{Analogy with liquid crystal systems}

Hartree-Fock calculations tend to overestimate the degree of ordering of a system. The influence of quantum and thermal fluctuations in the present system can be assessed to some degree by an analogy with two-dimensional (2d) liquid crystals. 20 The analogy is based on the fact that the UCDW shares its symmetry with $2 \mathrm{~d}$ smectic liquid crystals. The appropriate elastic variable of both systems is the phase $u$ of the density oscillations, $\delta \rho(\mathbf{r})=\rho_{0} \cos \left(q_{0} x-u(\mathbf{r})\right)$. In terms of $u$, the long-wavelength elastic free energy in the absence of forces tending to align the stripes reads 21

$$
F=\frac{A}{2} \int d \mathbf{r}\left\{\left(\frac{\partial u}{\partial x}\right)^{2}+\lambda^{2}\left(\frac{\partial^{2} u}{\partial y^{2}}\right)^{2}\right\}
$$

where $A$ is an elastic constant and $\lambda$ a length which is presumably of the order of the UCDW period. The absence of a term involving $(\partial u / \partial y)^{2}$ is a consequence of the global rotation symmetry of the system. (Note that $u=a y$ only leads to a global rotation of the CDW to linear order in $a$ and thus leaves the free energy unchanged.)

It is an immediate consequence of this elastic free energy that dislocations will cost only a finite energy 21 Thus there will be a finite density of dislocations at any non-zero temperature and the stripes should be broken down to stripe segments. This is expected to destroy translational long-range order but preserve quasi-long-

submitted to World Scientific on November 12, 1999 
range orientational order of the remaining stripe segments. Thus, the smectic order predicted by the HFA is preserved only at zero temperature. At non-zero temperatures, the system is analogous in terms of symmetries to a $2 \mathrm{~d}$ nematic liquid crystal.

As the temperature increases the system should undergo a Kosterlitz-Thouless transition from the nematic phase to an isotropic phase in which the orientational order of the stripe segments disappears. Nevertheless, short-range stripe ordering should still persist up to the presumably much higher Hartree-Fock transition temperature $T_{c}^{h f}$.

MacDonald and Fisher 9 have recently studied the stability of the UCDW against quantum fluctuations in the framework of the elastic theory and found that quantum fluctuations are not strong enough to destroy the smectic order predicted by the HFA.

While much can be learned about the influence of quantum and thermal fluctuations from this analogy, much less is known about the influence of disorder on the UCDW.22 One may expect that transport properties of the striped phases should be affected by even small amounts of disorder on the substrate, which will pin the stripe positions at low temperatures. Disorder should also lead to a finite density of dislocations, even at zero temperature. Moreover, since the forces aligning the stripes are believed to be very weak, steps or other large-scale features of the GaAs-AlGaAs interface may lead to large regions where the stripes are oriented differently from the average preferred direction.

\subsection{Transport properties}

An important problem is a quantitative understanding of transport in the striped phases. It had been previously noted in various context 23 that an externally imposed density modulation can lead to strongly anisotropic transport in the presence of a magnetic field. While this effect is indeed related to the development of anisotropic transport in higher Landau levels, none of the developed formalisms apply directly to the present system.

We start with the idealized case of a pinned unidirectional CDW near $\nu=$ $N+1 / 2$, first studied by MacDonald and Fisherg (cf. Fig. 11a). The stripes are taken parallel to the $y$ axis and the CDW has period $a$. Current can be carried by the internal edge channels belonging to the $(N+1)$ st LL which travel along each stripe edge, with velocity $v_{F}$ and density of states $N(0)=1 / h v_{F}$. Impurities lead to scattering between these edge channels. The scattering rates should decrease rapidly with increasing distance between edges. Thus, we include only scattering between neighboring edges and denote the scattering rate across electron stripes (stripes of filling factor $N+1$ ) as $1 / \tau_{e}$ and across hole stripes (stripes of filling factor $N$ ) as $1 / \tau_{h}$. Each scattering event acts as backscattering with regard to the $y$ direction and a single random-walk step in the $x$ direction. In addition, we assume that quantum interference does not play an important role. In this case, we can obtain the conductivity tensor via the Einstein relation from the classical diffusion constants.

The density of excitations on the up-moving and down-moving edge channels of

submitted to World Scientific on November 12, 1999 


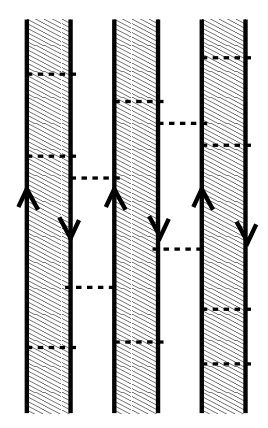

(a)

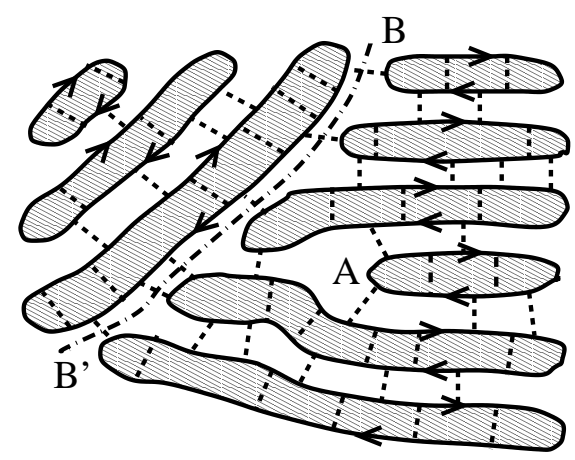

(b)

Figure 1. (a) Ideal and (b) realistic stripe structures. Shaded and unshaded regions represent stripes with filling factors $N+1$ and $N$, respectively. Arrows indicate the direction of electron flow on edge states. The dashed lines are scattering centers allowing electrons to tunnel between neighboring edge states. The realistic stripe structure contains a dislocation (A) and a large angle grain boundary $\left(\mathrm{B}-\mathrm{B}^{\prime}\right)$.

the $i$-th electron stripe, denoted by $P_{t}^{+}(y, i)$ and $P_{t}^{-}(y, i)$ respectively, satisfy the rate equations

$$
\begin{aligned}
& \left(\partial_{t}+v_{F} \partial_{y}\right) P_{t}^{+}(i)=-\left(\frac{1}{\tau_{e}}+\frac{1}{\tau_{h}}\right) P_{t}^{+}(i)+\frac{1}{\tau_{h}} P_{t}^{-}(i-1)+\frac{1}{\tau_{e}} P_{t}^{-}(i) \\
& \left(\partial_{t}-v_{F} \partial_{y}\right) P_{t}^{-}(i)=-\left(\frac{1}{\tau_{e}}+\frac{1}{\tau_{h}}\right) P_{t}^{-}(i)+\frac{1}{\tau_{e}} P_{t}^{+}(i)+\frac{1}{\tau_{h}} P_{t}^{+}(i+1) .
\end{aligned}
$$

Acting on Eq. (2) with $\partial_{t}-v_{F} \partial_{y}+\left(1 / \tau_{h}+1 / \tau_{e}\right)$, inserting Eq. (3), and dropping second-order time derivatives, one obtains the diffusion equation

$$
\partial_{t} P_{t}^{+}(i)=\frac{v_{F}^{2}}{2} \frac{\tau_{e} \tau_{h}}{\tau_{e}+\tau_{h}} \partial_{y}^{2} P_{t}^{+}(i)+\frac{1}{2\left(\tau_{e}+\tau_{h}\right)}\left[P_{t}^{+}(i+1)-2 P_{t}^{+}(i)+P_{t}^{+}(i-1)\right] .
$$

The same equation is satisfied by $P_{t}^{-}(i)$. One can now read off the diffusion constants

$$
D_{x x}=\frac{a^{2}}{2} \frac{1}{\tau_{e}+\tau_{h}} \quad D_{y y}=\frac{v_{F}^{2}}{2} \frac{\tau_{e} \tau_{h}}{\tau_{e}+\tau_{h}} .
$$

According to the Einstein relation, the conductivity is related to the diffusion constants by $\sigma_{\alpha \alpha}=e^{2}(2 / a)\left(1 / h v_{F}\right) D_{\alpha \alpha}$ and one obtains 9

$$
\sigma_{x x}=\frac{e^{2}}{h} \frac{a}{v_{F}\left(\tau_{e}+\tau_{h}\right)} \quad \sigma_{y y}=\frac{e^{2}}{h} \frac{v_{F}}{a} \frac{\tau_{e} \tau_{h}}{\tau_{e}+\tau_{h}} .
$$

To obtain the Hall conductivity, we note that the $N$ completely filled LL's contribute $N e^{2} / h$. To find the contribution of the partially filled topmost LL, we apply a chemical potential gradient in the $x$ direction. Assuming a chemical potential drop of $e v$ between the two edges of an electron stripe, we have a Hall current $j_{y}=\left(e^{2} / h\right)(v / a)$ in the $y$ direction and a diffusion current $j_{x}=\left(e / \tau_{e}\right)\left(1 / h v_{F}\right) e v$ 
in the $x$ direction. Comparing with $j_{x}=\sigma_{x x} E_{x}$, we find $v=\left[\tau_{e} /\left(\tau_{e}+\tau_{h}\right)\right] E_{x} a$ and therefore a Hall conductivity off

$$
\sigma_{x y}=\frac{e^{2}}{h}\left(N+\frac{\tau_{e}}{\tau_{e}+\tau_{h}}\right) .
$$

Remarkably, these results make a number of predictions which are independent of the microscopic parameters. 10 Following MacDonald and Fisher we first focus on the symmetric point $\tau_{e}=\tau_{h} \equiv \tau$. If one assumes particle-hole symmetry in the partially filled LL, this would correspond to half filling of the topmost Landau level, $\nu=N+1 / 2$. In this case, we deduce from Eq. (6) that the product of the diagonal conductivities takes on a universal value 9

$$
\sigma_{x x} \sigma_{y y}=\left(e^{2} / 2 h\right)^{2},
$$

independent of the period, Fermi velocity, or the scattering rate. Likewise, one finds that the Hall conductivity becomes independent of the microscopic parameters, $\sigma_{x y}=\frac{e^{2}}{h}(N+1 / 2)$. Hence, we can also rewrite the product rule in terms of the diagonal resistivities as

$$
\rho_{x x} \rho_{y y}=\left(h / e^{2}\right)^{2} \frac{1}{\left[N^{2}+(N+1)^{2}\right]^{2}} .
$$

For the Hall resistivity at the symmetric point one finds $\rho_{x y}=\left(h / e^{2}\right)(2 N+1) /\left[N^{2}+\right.$ $\left.(N+1)^{2}\right]$.

While the product of the diagonal resistivities is universal, the anisotropy $\rho_{x x} / \rho_{y y}$ depends on the microscopic parameters,

$$
\frac{\rho_{x x}}{\rho_{y y}}=\left(\frac{v_{F} \tau}{a}\right)^{2}
$$

and is given by the square of the ratio of the basic diffusion steps in the $y$ and $x$ directions.

It is also interesting to note that if we assume that the diagonal conductivity (or, equivalently, the scattering rates $1 / \tau_{e}$ and $1 / \tau_{h}$ ) do not depend significantly on the Landau level index $N$, say at the symmetric point, then the resistivities decrease with increasing Landau level index roughly as $1 / N^{2}$. This seems to be in rather good agreement with the experimental results for the peak heights in the high-resistance direction.

Initial results indicate that the product rule Eq. (9) is reasonably well satisfied in experiment 24 This may be surprising in view of the expectation that the experimental samples should be quite far from the perfect stripe ordering assumed here. Thus, experiment raises the question whether the product rule is not in fact valid much more generally than indicated by this derivation. An additional question is related to the neglect of quantum interference. Since the experimental anisotropy in the resistivity is about five, 14 these results [cf. Eq. (10)] would imply that the electrons hop between edges after traveling only a distance of a few cyclotron radii along the edge. For such a situation, quantum interference effects should be important, particularly since the experiments are performed at extremely low temperatures. While it is currently not known how quantum interference affects

submitted to World Scientific on November 12, 1999 
the validity of the product rule, it is certainly possible that it leads to significant deviations.

Transport in more realistic stripe structures has been studied by von Oppen et al 10 The starting point is that the conductivity tensor of the perfect stripe structure actually satisfies an even more general relation, namely the semicircle lau 10

$$
\sigma_{x x} \sigma_{y y}+\left(\sigma_{x y}-\sigma_{h}^{0}\right)^{2}=\left(e^{2} / 2 h\right)^{2},
$$

with $\sigma_{h}^{0}=\frac{e^{2}}{h}(N+1 / 2)$, which holds also away from the symmetric point. This relation can be generalized to a wide class of stripe structures. In particular, a semicircle law holds even in the presence of topological and orientational defects such as dislocations and grain boundaries if one assumes that the defects are pinned by disorder. An example of such a more general stripe structure is shown in Fig. 1(b).

Strictly speaking, one finds that the macroscopic conductivity tensor $\hat{n}^{*}$ (relating the spatially averaged currents and fields) satisfies the semicircle law 10

$$
\sigma_{1}^{*} \sigma_{2}^{*}+\left(\sigma_{h}^{*}-\sigma_{h}^{0}\right)^{2}=\left(e^{2} / 2 h\right)^{2} .
$$

Here, the macroscopic conductivity tensor $\hat{\sigma}^{*}=\hat{\sigma}_{d}^{*}+\sigma_{h}^{*} \hat{\epsilon}$ is written as the sum of its dissipative part $\hat{\sigma}_{d}^{*}$ and Hall component $\sigma_{h}^{*} \hat{\epsilon}$ with $\hat{\epsilon}$ the totally antisymmetric tensor. $\sigma_{1}^{*}$ and $\sigma_{2}^{*}$ are the eigenvalues of the (real symmetric) dissipative part $\hat{\sigma}_{d}$.

The product rule Eq. (8) is a special case of the semicircle law for the symmetric point $\sigma_{x y}=\left(e^{2} / h\right)(N+1 / 2)$. Thus, the more general validity of the semicircle law also implies the same for the product rule, thereby explaining its agreement with experiment. Moreover, this makes the experimental results consistent with a picture where electrons hop between edges much more rarely, while the anisotropy is reduced by the presence of defects such as dislocations and grain boundaries. In such a picture, neglecting quantum interference may indeed be justified.10

The geperalized semicircle law Eq. (12) can be supported by two different arguments 10 The most general derivation uses results obtained by Shimshoni and Auerbach 25 for a model of the "quantized Hall insulator." In this argument, one maps the problem to a network of puddles of filling factor $\nu=1$ in vacuum. Neglecting quantum interference, assuming that the network is planar, and that the stripe structure is pinned, it was shown by Shimshoni and Auerbach that the corresponding (properly defined) Hall resistance equals $R_{x y}=h / e^{2}$. Following the mapping from the stripe structure to the puddle network in reverse, one finds that this implies the semicircle law (12) 10 The assumption that the network is planar would for example be violated if one included next-to-nearest neighbor hopping between edges.

Alternatively, one can also give a continuum argument 10 for the semicircle law Eq. (12) based on a duality transformation first exploited by Dykhne and Ruzin.26 In this argument, one assumes that the defect density is sufficiently low so that one can define a local conductivity tensor which everywhere satisfies the semicircle relation (11). The defects lead to spatial variations of the scattering rates and of the principal axes of the dissipative part of the conductivity tensor. Using a duality transformation, involving new currents and fields which are linear combinations of the original currents and fields, and choosing the dual system to be the time reverse

submitted to World Scientific on November 12, 1999 
of the original one, it can then be shown 10 that the macroscopic conductivity tensor satisfies the semicircle law Eq. (12).

Several authors observed that the internal edge modes should be Luttinger liquids.20 It has been argued that the stripe phase is unstable (possibly towards an anisotropic Wigner crystal) due to backscattering, albeit only below experimentally accessible temperatures. MacDonald and Fisher have also suggested that the Luttinger liquid behavior may explain the experimentally observed nonlinearities in the diagonal resistivity. Within the transport theory sketched above, Luttinger liquid correlations imply that the scattering rates $1 / \tau_{e}$ and $1 / \tau_{h}$ decrease with increasing voltage. Thus, the anisotropy increases with increasing voltage (or applied current), as was observed in experiment. However, there are not yet detailed predictions for the full temperature and current dependences which might be compared with experiment.

Fradkin et al.27 have used the liquid-crystal analogy to study the temperature dependence of the anisotropy for the transition from the nematic to the isotropic phase. They argue on the basis of symmetry that close to $T_{c}$ the anisotropy should be proportional to the order parameter of an xy model with director order parameter in the presence of a small background anisotropy. Using Monte-Carlo results for this model they attempt to fit the experimental anisotropy, using two free parameters. The validity of the analysis is hard to assess, however, because it is unknown for which range around $T_{c}$ the assumed proportionality holds.

\section{Conclusions and open questions}

The discovery of the anisotropic phases in higher Landau levels 12 , combined with the earlier theoretical predictions of CDW states 6 in this filling factor range, has opened a new chapter of quantum Hall physics. Initially, the experimental evidence for charge density wave ordering has been purely qualitative. The product ruld 9 and the semicircle law 10 discussed in Sec. 3.3 are the first predictions for the striped CDW phases which can be tested quantitatively in experiment. Initial results seem to indicate reasonable agreement.24

It seems clear that the investigation of the new charge density wave states in higher Landau levels has only started and much interesting physics remains to be studied. Obvious open questions include the following: The mechanism which causes the stripes to line up preferentially with a particular axis of the GaAs substrate is not well understood. The influence of disorder on the striped phases has not been studied. There is currently no explanation for the observation that various quantities have a prominent dependence on whether the Fermi energy is in the lower or upper spin component of a LL. The influence of quantum interference on transport has not been studied. Filling factors away from half filling of the topmost LL have received relatively little attention.

\section{Acknowledgments}

We benefitted from discussions with J. Eisenstein, B. Huckestein, M. Shayegan, S. Simon, D. Shahar and H. Stormer. The work was supported in part by SFB

submitted to World Scientific on November 12, 1999 
341, NSF grant DMR-94-16910, US-Israel BSF grant 98-354, DIP-BMBF grant, a Minerva foundation grant, and the Israeli Academy of Science.

\section{References}

1. M.P. Lilly, K.B. Cooper, J.P. Eisenstein, L.N. Pfeiffer, and K.W. West, Phys. Rev. Lett. 82, 394 (1999).

2. R.R. Du, D.C. Tsui, H.L. Stormer, L.N. Pfeiffer, K.W. Baldwin, and K.W. West, Solid State Comm. 109, 389 (1999).

3. M.M. Fogler, A.A. Koulakov, and B.I. Shklovskii, Phys. Rev. B 54, 1853 (1996); A.A. Koulakov, M.M. Fogler, and B.I. Shklovskii, Phys. Rev. Lett. 76, 499 (1996).

4. R. Moessner, J.T. Chalker, Phys. Rev. B 54, 5006 (1996).

5. R.B. Laughlin, Phys. Rev. Lett. 50, 1395 (1983).

6. H. Fukuyama, P.M. Platzman, and P.W. Anderson, Phys. Rev. B 19, 5211 (1979).

7. E.H. Rezayi, F.D.M. Haldane, and K. Yang, Phys. Rev. Lett. 83, 1219 (1999).

8. M. Shayegan and H.C. Manoharan, cond-mat/9903405.

9. A.H. MacDonald and M.P.A. Fisher, cond-mat/9907278.

10. F. von Oppen, B.I. Halperin, and A. Stern, cond-mat/9910132.

11. W. Pan, R.R. Du, H.L. Stormer, D.C. Tsui, L.N. Pfeiffer, K.W. Baldwin, and K.W. West, cond-mat/9903160.

12. M.P. Lilly, K.B. Cooper, J.P. Eisenstein, L.N. Pfeiffer, and K.W. West, condmat/9903196.

13. J.P. Eisenstein, M.P. Lilly, K.B. Cooper, L.N. Pfeiffer, and K.W. West, condmat/9909238.

14. S.H. Simon, cond-mat/9903086.

15. M.P. Lilly, K.B. Cooper, and J.P. Eisenstein, cond-mat/9903153.

16. K.B. Cooper, M.P. Lilly, J.P. Eisenstein, L.N. Pfeiffer, and K.W. West, condmat/9907374.

17. I.L. Aleiner and L.I. Glazman, Phys. Rev. B 52, 11296 (1995).

18. T. Jungwirth, A.H. MacDonald, L. Smrcka, and S.M. Girvin, condmat/9905353.

19. T. Stanescu, I. Martin, and P. Philipps, cond-mat/9905116.

20. E. Fradkin, S.A. Kivelson, Phys. Rev. B 59, 8065 (1999).

21. D.R. Nelson, in Phase Transitions and Critical Phenomena, ed. by C. Domb and J.L. Lebowitz (Academic Press, London, 1983).

22. See, however, L. Radzihovsky and J. Toner, Phys. Rev. B 60, 206 (1999) for a recent study of smectic liquid crystals in the presence of disorder.

23. See, e.g., G.R. Aizin and V.A. Volkov, Sov. Phys. JETP 65, 188 (1987); and F. von Oppen, A. Stern, and B.I. Halperin, Phys. Rev. Lett. 80, 4494 (1998) and references therein.

24. J. Eisenstein, private communication and cond-mat/0003405 (to appear in Physica E).

25. E. Shimshoni and A. Auerbach, Phys. Rev. B 55, 9817 (1997).

26. A.M. Dykhne and I.M. Ruzin, Phys. Rev. B 50, 2369 (1994).

submitted to World Scientific on November 12, 1999 
27. E. Fradkin, S.A. Kivelson, E. Manousakis, and K. Nho, cond-mat/9906064.

submitted to World Scientific on November 12, 1999 